\title{
Design of Machine Vision Defect Detecting System Based on Halcon
}

\author{
Bin $\mathrm{Xu}^{\mathrm{a}}$, Wenbo $\mathrm{Ye}^{\mathrm{b}}$ and Yurong Wang ${ }^{\mathrm{c}}$ \\ A College of Automation, Wuhan University of Technology, Wuhan 430070, China. \\ a244524405@qq.com, bywb6113@gmail.com, c806277525@qq.com
}

Keywords: machine vision; defect detecting; Industry4.0; Intelligent manufacturing.

\begin{abstract}
It can be predicted that Industry4.0 is the general trend of mechanized integrated production in the future. In the field of Industry4.0, people have begun to focus on machine vision inspection. A defect detection machine vision system based on image processing software called Halcon is designed in this paper, which is designed to detect the bad spots on the screen in the production line, the defects of the PCB circuit board on the production line, as well as the scratches and cracks of the ceramic tiles on the production line. This design can be used to eliminate inferior products and strictly control the output rate of finished products.
\end{abstract}

\section{Introduction}

The system is designed to detect and test the following three scenarios:

1. screen bad point or bright spot detection.

2. circuit board defect detection (missing welding, leakage welding, device location inversion).

3. Tile classification and defect detection (cracking, scratching, etc.).

The system can identify and match the standard samples and test samples, and then display the results and transfer the results into the database or transfer the related signals to the PLC and the relevant executive agencies, so that the secondary product is released from the pipeline to ensure the production rate of the production line.

The overall system diagram of the system is shown in the FIG.1.The system consists of 5 parts: image acquisition unit, image processing unit, execution unit, communication unit and human-machine interaction unit. Next, we will briefly introduce the image acquisition unit and emphasis on the image processing algorithm.

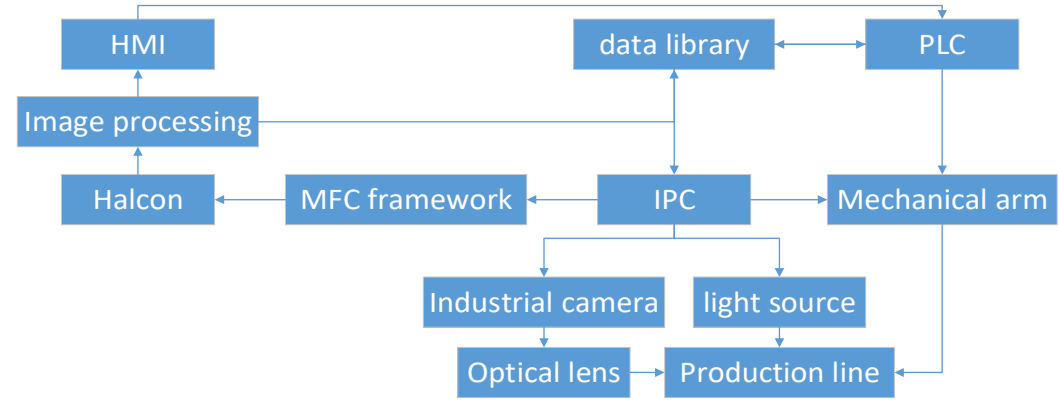

Figure 1. Overall system block diagram.

\section{Image Acquisition Unit}

According to the characteristics of the ceramic tile production line, this system has designed the light field for the production line, including the type of light source used, the selection of optical lens and industrial camera, and the installation position of each component on the production line.The schematic diagram of the light field is shown in the FIG.2. 


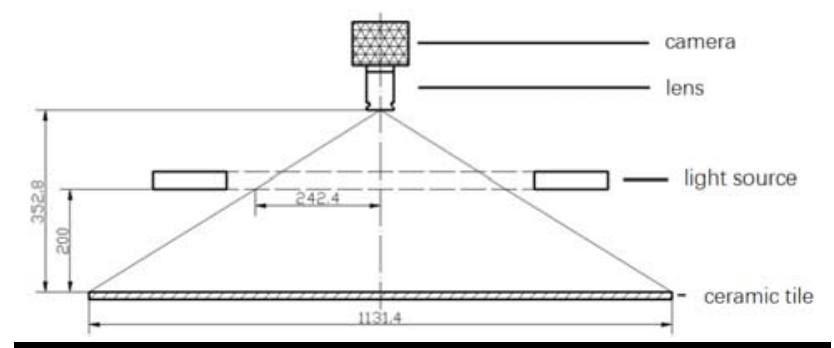

Figure 2. Schematic diagram of system light field design.

In this paper, we choose a LED light source with longer life and more stable performance and use a ring light source to obtain a bright light field, which determines the lighting angle according to different products. As for the lens, the $8 \mathrm{~mm}$ fixed focus lens are used in this paper to get a sufficient perspective to detect the larger tiles.

The industrial camera used in this system has an industrial camera with 1/3-inch imaging size and CCD sensor. The larger imaging size also ensures that the camera can capture the complete image of the product when it is kept at a distance from the detected product. The integrity of image processing is guaranteed. At the same time, the camera can shoot $30 \mathrm{fps}, 1280 * 960$ specifications of the video, which can be output in three video formats: RGB32, Y800 and Y16.

\section{Design of Image Processing Algorithm}

HALCON comes from academia, which is different from commercial software packages in the market. In fact, this is a set of images processing library, consisting of more than 1000 separate functions and the underlying data management core. It contains various kinds of basic geometry and image computing functions such as filtering, mathematical transformation, computational analysis, correction, classification and identification, shape search and so on.

This article will design the algorithm for the following three modules: screen bad point detection, PCB defect detection and ceramic tile crack detection.

\subsection{Algorithm of Screen Bad Point Detection}

The screen of the sample is divided into black and white. Under the black background, if the screen is qualified, there is no red point; if the screen is not qualified, red points exist. The screen bad point can be segmented by threshold segmentation and the location and size of the screen can be determined. The pattern of white is similar. The flow block diagram is shown in FIG.3.

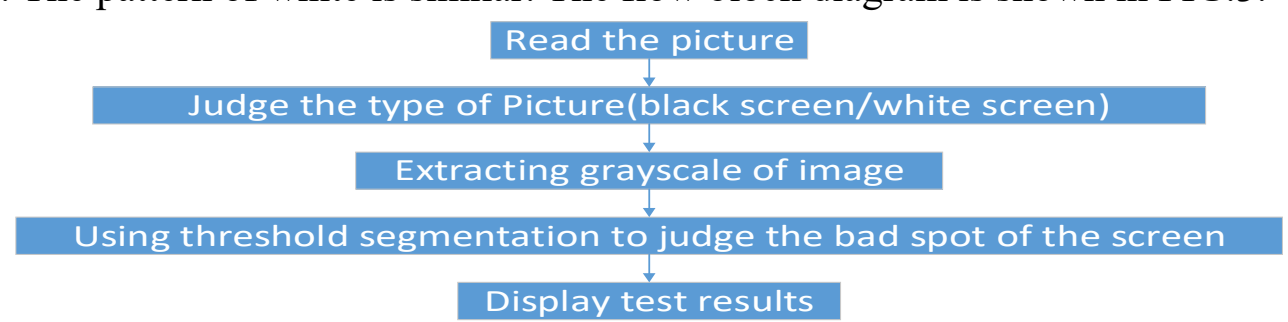

\subsection{Algorithm of PCB Defect Detection}

Figure 3. Flow chart of screen bad point detection program.

This design has done the following 3 kinds of defect detection. The flow block diagram is shown in FIG.4.

Chip pin short circuit detection: The short circuit of the chip pin is caused by too much soldering, resulting in short circuiting of some adjacent pins. Because the solder is too much and the short circuit is bright white, the bright pixels can be segmented by threshold segmentation, then the connectivity analysis is carried out to calculate the area of the block of continuous bright pixels. If the area is larger than a certain value, the solder is too much and the pin is short circuited.

Resistance leakage detection: the chip resistor is a black rectangular element, and the surface color of the circuit board is green. If the resistance is leaking, the center of the 2 pads is green. If the resistance is welded normally, the center is black rectangle. Based on this, the pixel color of each welding disc center is analyzed to determine whether the resistance of the spot is welded. 
Capacitance angle detection: the capacitance is the black cylindrical element. The black pixel is locked by the threshold segmentation. Then the relative larger area is selected through the connectivity analysis, which is defined as the capacitance area. Finally, the direction field is calculated and the angle of the capacitance is calculated.

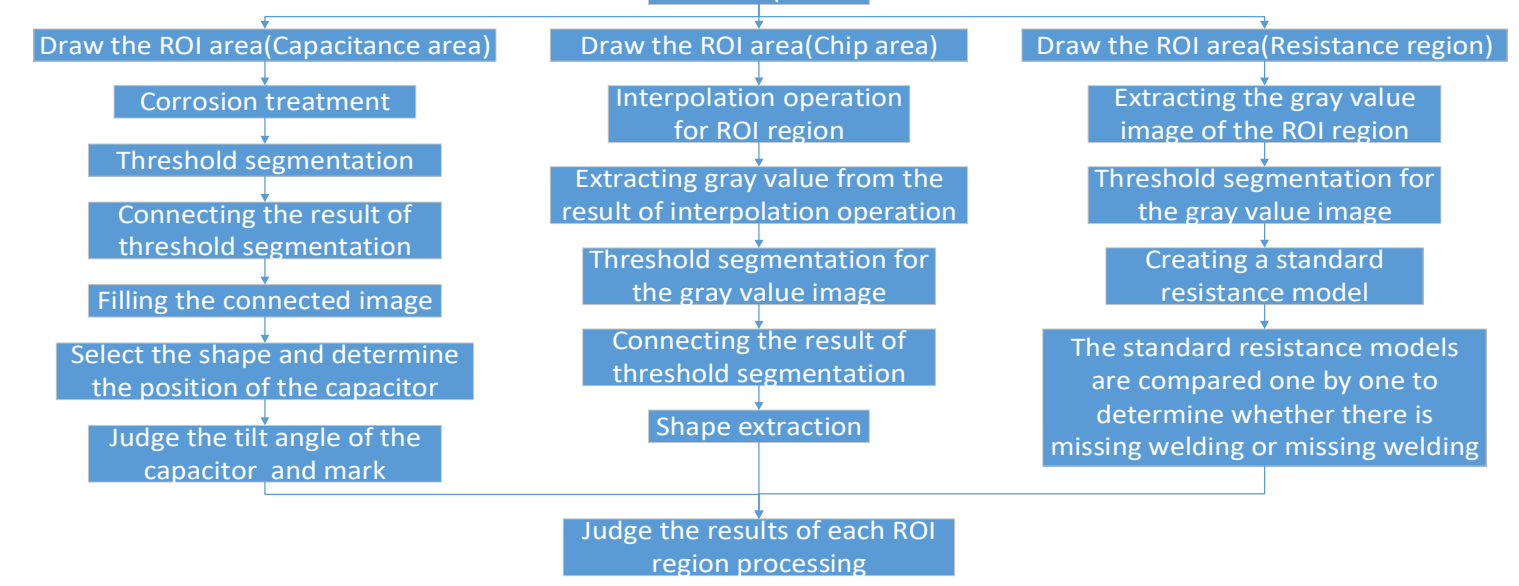

Figure 4. Flow chart of algorithm for PCB.

\subsection{Detection Algorithm for Ceramic Tile Crack / Scratch}

The sample tiles are light brown with irregular dark brown texture. Defects are divided into dark grey cracks and white scratches. One of the difficulties of the detection algorithm is to distinguish the defect from the texture of the tile itself. The texture of the tile itself is similar to the shape of the crack, but the color is distinctly different, so it can start with the color.

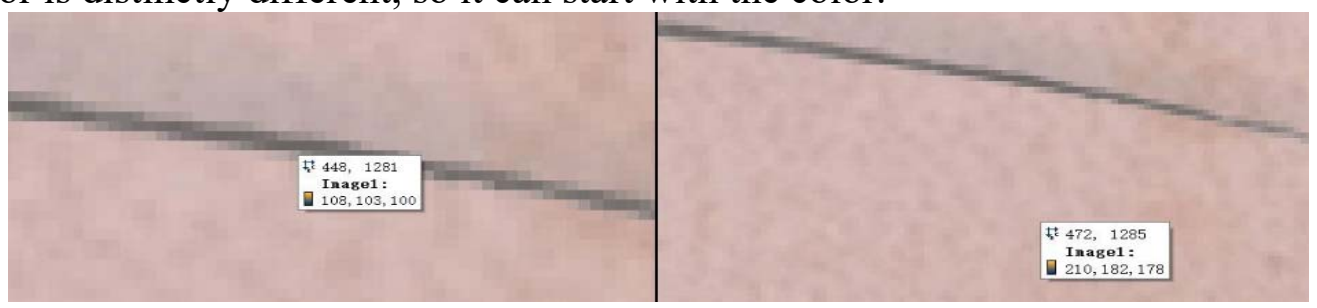

Figure 5. Pixel RGB value of tile.

As shown in the FIG.5, the pixels in the crack are RGB (108103100), the normal area is RGB (210182178), the pixel value of the RGB channel at the crack is close to $1: 1: 1$, and the size is about 100 , and the $\mathrm{R}$ channel of the normal region pixel value is obviously higher than the other 2 channels. Through these characteristics we can divide the pixels in the crack. And use the red pixels to illuminate, according to the number of pixels to determine whether the tile is qualified or unqualified. The flow block diagram is shown in FIG.6.

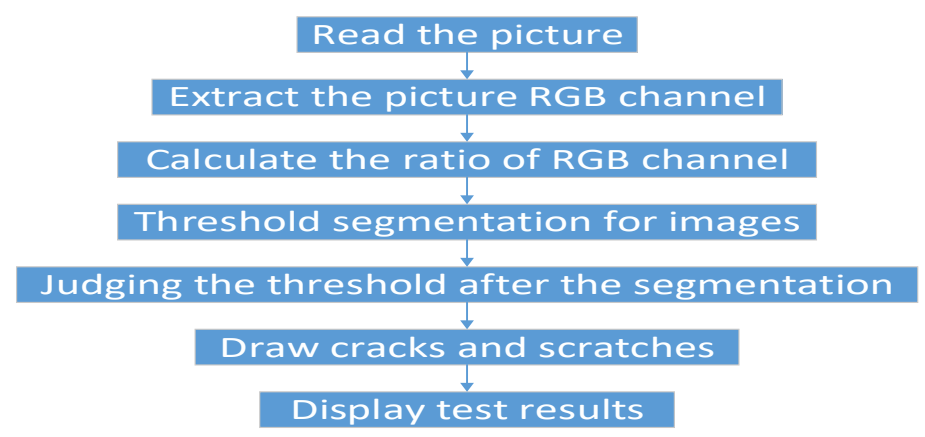

Figure 6. Flow chart of algorithm for ceramic tile crack / scratch. 


\section{Test and Result Display}

\subsection{Environment Construction}

This project is initially configured on Visual Studio2010, and the library files used are OpenCV2.4.9 and Halcon12 respectively. Due to the development and debugging needs, the project will be transplanted to Visual Studio2017 under the Windows10 system for debugging.

MFC is the abbreviation of Microsoft Foundation Class Library. It is a C++ class library implemented by Microsoft Corporation. It mainly encapsulates most of the windows API functions. MFC adopts an object-oriented programming(OOP) method. It has the advantages of easy maintenance, high quality, high efficiency and easy expansion. In combination with the advantages of $\mathrm{C}++$ and $\mathrm{MFC}$, we have configured the hybrid programming for MFC and Halcon. On this basis, we design the Human-computer interaction interface in the resource file of MFC project.

\subsection{Hardware Test}

The rate of the industrial ceramic tile production line is $60-200$ pieces per minute, which means the video stream refresh frequency of the system is supposed to be $4 \mathrm{~Hz}$, that is to say, 4 pictures need to be processed at the fastest time per second. In the software testing scheme of this system, each tile defect detection takes about $141 \mathrm{~ms}$, far less than $250 \mathrm{~ms}$, so it can theoretically meet the fastest flow speed of the industrial production line.

\subsection{The Display of the Results}

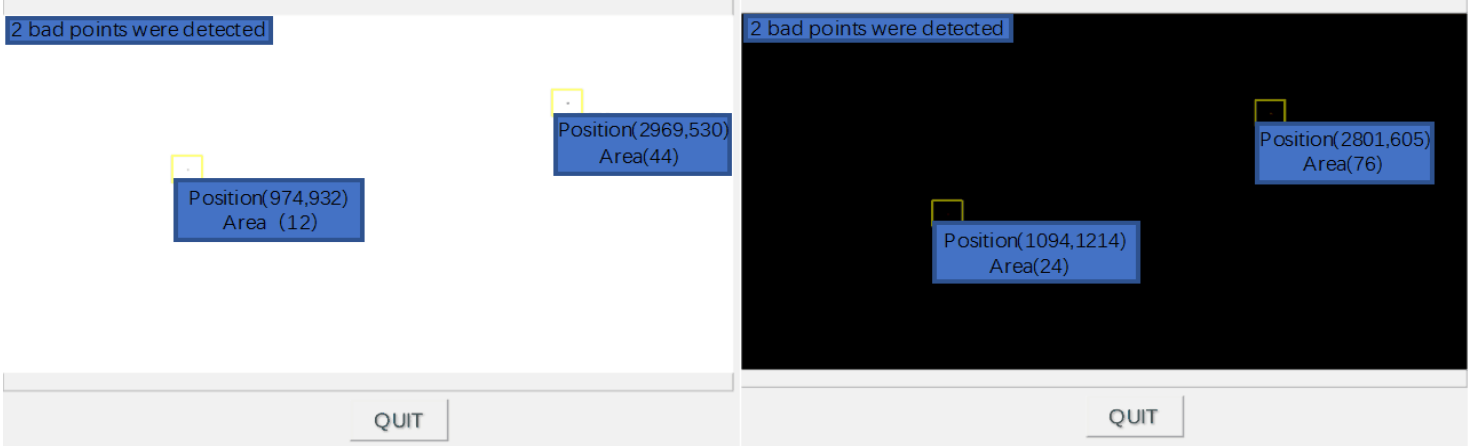

Figure 7. Result of screen bad point detection.

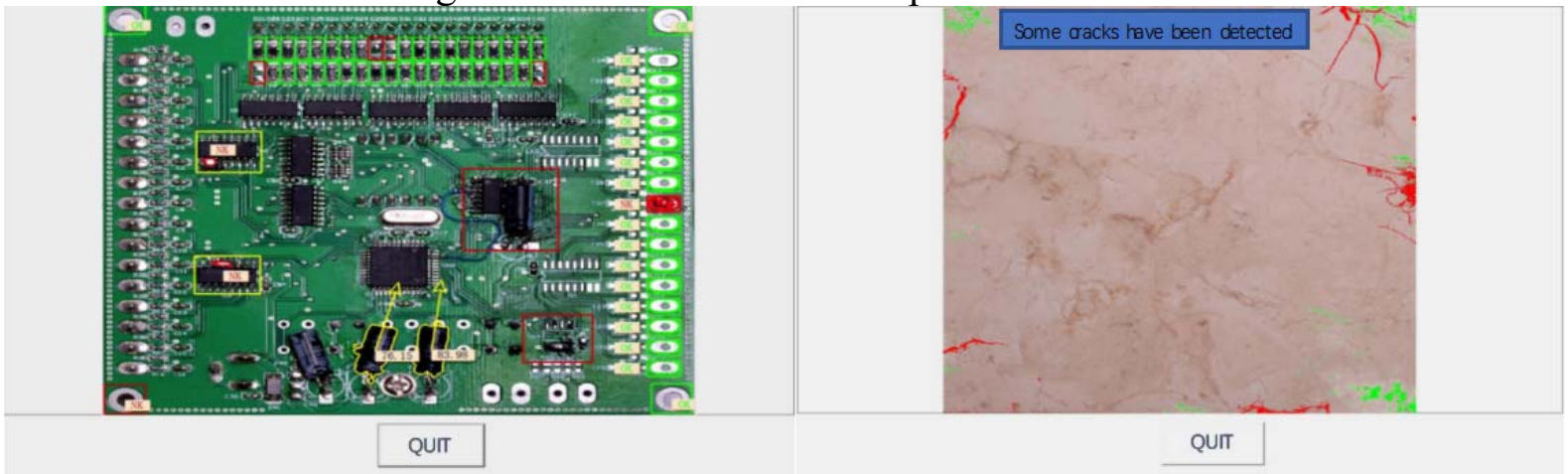

Figure 8. Result of PCB and ceramic tile defect detection.

The result of screen bad point detection is shown in FIG.7. Whether white background or black background, we can detect the bad points and get the location and area of the bad points. The result of PCB and ceramic tile defect detection are shown in FIG.8. The system can detect defects of chip pins, capacitors and resistors accurately. Similarly, the system can also accurately detect cracks and scratches on tiles.

\section{Conclusion}

A defect detection machine vision system based on image processing software called Halcon is designed in this paper, which is designed to detect the bad spots on the screen in the production line, the defects of the PCB circuit board on the production line, as well as the scratches and cracks of the ceramic tiles on the production line. Through the collation and comparative analysis of multi-party 
data, this paper designs an image acquisition unit which is conducive to the high-quality imaging of the system. Most important of all, most importantly, based on the advantages of $\mathrm{C}++$ and MFC, this paper designs an efficient image processing algorithm based on MFC and Halcon mixed programming configuration. After testing, each tile defect detection takes about $141 \mathrm{~ms}$. The effectiveness of the proposed algorithm is verified through the Visual Studio 2017 integrated development environment.

\section{Acknowledgments}

Funding from the Keboda Foundation is gratefully acknowledged. The author wishes to express the great gratitude to Mr. Xiaoqiang Xu, Professor of Wuhan University of Technology, who gave strong technical support in this study.

\section{References}

[1]. Mohammad H. Karimi and Davud Asemani, "Surface defect detection in tiling industries using digital image processing methods: Analysis and evaluation," in ISA Transactions (Volume 53, Issue 3), 2014, pp.834-844.

[2]. M.R. Xue and K.M. Li, "Application of Halcon in Digital Image and Signal," in Applied Mechanics and Materials (Volumes 716-717),2014, pp.1338-1340.

[3]. Tastimur and Canan, "Rail Defect Detection and Classification with Real Time Image Processing Technique," in International Journal of Computer Science and Software Engineering (Volume 5, Issue 12), 2016, pp.283-290.

[4]. Junjie Hou, Deep into the MFC (Huazhong University of Science and Technology Press, Wuhan,2001), pp.447-462.

[5]. Xia Yan and Jianqiang Niu, "Camera calibration based on HALCON software," in digital technology and application, 2010, pp.112-113.

[6]. Qiong Zhang, "Image quality detection of unmarked printed matter based on HALCON," in Journal of Shantou University (NATURAL SCIENCE EDITION),2011, pp.63-68. 